\title{
Periodontal Status of Patients with Non -Insulin Dependent Diabetes Mellitus
}

\author{
Dr. Sameer Aryal, ${ }^{1}$ Dr. Binod Adhikari, ${ }^{1}$ Dr. Reshu Agrawal, ${ }^{2}$ \\ Dr. Krishna Gopal Bhuju, ${ }^{1}$ Dr. Riwas Karki ${ }^{1}$ \\ ${ }^{1}$ Department of Dentistry, Nepalese Army Institute of Health Sciences (NAIHS), Shree Birendra Hospital (SBH), Kathmandu, Nepal; \\ ${ }^{2}$ School of Public Health, Patan Academy of Health Sciences, Lalitpur, Nepal.
}

\begin{abstract}
Introduction: Association of Diabetes Mellitus and periodontal disease has been well established over the last few decades. It is a well established risk factor in the pathogenesis of periodontal disease.

Objective: This study was conducted to assess the prevalence of periodontal disease in non-insulin dependent diabetes mellitus.

Methods: Individuals with non-insulin dependent diabetes mellitus for the duration of at least five years were selected. Plaque Index (PII), Gingival Index (GI), Loss of attachment (LA) and Probing Depth (PD) were recorded using William's Periodontal probe.

Results: Patients with Non-Insulin Dependent Diabetes Mellitus demonstrated higher loss of attachment.

Conclusion: The results showed statistically significant association between non-insulin dependent diabetes mellitus and periodontal disease thus supporting it as a risk factor for periodontitis.

Keywords: Attachment loss; index; non-insulin dependent diabetes mellitus; periodontitis; probing.
\end{abstract}

\section{INTRODUCTION}

Periodontal Disease is an entity of chronic infection, which involves the supporting structures of teeth. There is extensive evidence that this infection is associated with various local and systemic factors. Studies have shown that individuals with diabetes have more gingival inflammation than individuals without. $^{1}$ Epidemiologic research have shown that diabetes mellitus increases the risk and severity of periodontal diseases.-4 Many previous studies have shown that individuals with controlled diabetes exhibit less severe periodontitis than the ones whose disease are not in control. ${ }^{7-12}$ There exists a strong association with poorly controlled diabetes mellitus thus making periodontal disease the "sixth complication of diabetes." ${ }^{13}$ American Diabetes Association has officially recognised that periodontal disease

\section{Correspondence:}

Dr. Sameer Aryal

Department of Dentistry, Nepalese Army Institute of Health Sciences (NAIHS), Shree Birendra Hospital (SBH), Kathmandu, Nepal.

email: dentistnepal@yahoo.com

\section{Citation}

Aryal S, Adhikari B, Agrawal R, Bhuju KG, Karki R. Periodontal Status of Patients with Non-Insulin Dependent Diabetes Mellitus. J Nepal Soc Perio Oral Implantol. 2019;3(5):2-5. is commonly observed in diabetes patients. This study was conducted to assess the prevalence and status of periodontal disease in noninsulin dependent diabetes mellitus.

\section{METHODS}

For the purpose of this study 86 individuals with diabetes were conveniently selected from the diabetes clinic that runs at Shree Birendra Hospital, Chhauni, Kathmandu, Nepal. Consent was taken from all patients to use the data without exposing their individual identification. All the subjects selected were Non-Insulin Dependent Diabetes Mellitus for the duration of at least five years. The diabetes clinic runs twice a week at the hospital and 20 to 25 cases were selected for examination for this study. In this way this cross-sectional study was completed with the duration of the study of three weeks period. Few variables were considered when selecting the study subjects. Both the sexes and various age groups were taken with maximum being up to 70 years.

Consultant periodontist was responsible for examination using a William's periodontal probe. To limit the biasness one consultant conducted all the examination. Following parameters were recorded during the examinations:

Silness and Loe Plaque Index (PI, 1964): The thickness of plaque at the cervical margin of the tooth closest to the gums 
were assessed. All four surfaces were examined: Distal, Mesial, Lingual, Buccal of 16, 12, 24, 36, 32, and 44. Scoring Criteria: 0 $=$ No Plaque; $1=$ A film of plaque adhering to the free gingival margin and adjacent area of tooth the plaque may be seen in situ only after application of disclosing solution or by using probe on tooth surface; 2 = Moderate accumulation of soft deposits within the gingival pocket, or the tooth and gingival margin which can be seen with the naked eye; 3 = Abundance of soft matter within the gingival pocket and/or on the tooth and gingival margin.

To calculate PI, 0 to 3 scores for each surface were given. Plaque index for a tooth: Scores were added and then divided by four. Plaque index for group of teeth: Scores for individual teeth were added and then divided by number of teeth. Plaque index for the individual: Indices for each of the teeth were added and then divided by the total number of teeth examined. Plaque index for group: All indices are taken and divided by number of individuals. The PI scores were interpreted as: $0=$ Excellent; 0.1-0.9 = Good; 1.0-1.9 = Fair; 2.0-3.0 = Poor plaque control.

Loe and Silness Gingival Index (GI, 1963): One of the most widely accepted and used gingival indices. GI assess the severity of gingivitis and its location in four possible areas. Mesial, Lingual, Distal, Facial, Only qualitative changes are assessed. For GI scoring, all surfaces of the index teeth 16, $12,24,36,32$, and 44 were examined. The teeth and gingiva were first dried with a blast of air and/or cotton rolls. The tissues are divided into four gingival scoring units: distofacial papilla, facial margin, mesiofacial papilla and entire lingual margin. A blunt periodontal probe was used to assess the bleeding potential of the tissues. Following scoring criteria were used: $0=$ Absence of inflammation/normal gingiva; $1=$ Mild inflammation, slight change in colour, slight oedema, no bleeding on probing; 2 = Moderate inflammation, moderate glazing, redness, oedema and hypertrophy.bleeding on probing; 3 = Severe inflammation, marked redness and hypertrophy, ulceration, tendency to spontaneous bleeding.
The GI score for the tooth was calculated by totaling the scores around each tooth and dividing by the number of surfaces per tooth examined. Totaling all of the scores per tooth and dividing by the number of teeth examined gave the gingival index score for individual. The GI scores were interpreted as: $0.1-1.0=$ Mild gingivitis; $1.1-2.0=$ Moderate gingivitis; 2.1 $3.0=$ Severe gingivitis .

Loss of attachment (LOA): When recession of the gingival margin is present, the LOA is calculated by adding the probing depth with the distance of cementoenamel junction (CEJ) to gingival margin level. When the gingival margin is coronal to the CEJ, the LOA is calculated by subtracting the distance of gingival margin level to CEJ from the probing depth. When gingival margin was at CEJ, the LOA was calculated as being equal to probing depth.

Probing Depth (PD): In a healthy mouth, a probing depth can be anywhere from 1-3 millimeters deep. To measure a pocket periodontal probe was used. With the help of the William's periodontal probe we can measure from the crest of the marginal gingiva to the base of the pocket in millimeter. The bottom of the pocket is the area where the tissue is connected through ligaments to the root.

\section{RESULTS}

Table 1 shows that majority (51.2\%) of the patients belonged to the age group of 41 to 60 years ( $58.27 \pm 11.09$ years). More than half (61.6\%) of the study participants were male.

In the current study, more than half of the individuals with diabetes had fair to poor plaque index scores with mean of 1.19. All the patients suffered from some form of gingivitis with almost one tenth showed severe gingivitis. More than ninety percent showed gingival recession. Loss of attachment was noticed in almost 95\% of study participants with high average value of $3.27 \mathrm{~mm}$. Similarly, average probing depth among study participants was $2.39 \mathrm{~mm}$. Thus, we can observe a high prevalence of periodontal disease among the diabetes study individuals (Table 2).

Table 1: Age and sex distribution of the study patients $(n=86)$.

\begin{tabular}{|c|c|c|c|}
\hline Demographic variables & Categories & $\mathrm{n}(\%)$ & Mean \pm S.D. \\
\hline \multirow{2}{*}{ Age } & $20-40$ years & $5(5.8)$ & $58.27 \pm 11.09$ years \\
\hline \multirow{2}{*}{ Sex } & $41-60$ years & $44(51.2)$ & \\
\hline & $61-80$ years & $37(43)$ & \\
\cline { 2 - 3 } & Male & $53(61.6)$ & \\
\hline
\end{tabular}


Table 2: Prevalence of periodontal disease among the study participants $(n=86)$.

\begin{tabular}{|l|c|c|c|}
\hline \multicolumn{1}{|c|}{ Demographic variables } & Categories & $\mathrm{n}(\%)$ & Mean \pm S.D. \\
\hline \multirow{3}{*}{ Plaque Index } & Good & $39(45.3)$ & $1.19 \pm 0.54$ \\
\hline \multirow{2}{*}{ Gingival Index } & Fair & $39(45.3)$ & $8(9.3)$ \\
\hline \multirow{2}{*}{ Gingival Recession (in mm) } & Poor & $27(31.4)$ & $1.34 \pm 0.61$ \\
\hline \multirow{2}{*}{ Loss of Attachment (in mm) } & Moderate & $51(59.3)$ & $0.88 \pm 0.52$ \\
\hline \multirow{2}{*}{ Probing Depth (in mm) } & Poor & $8(9.3)$ & $3.27 \pm 1.42$ \\
\hline
\end{tabular}

Table 3 shows that more than $90 \%$ of patients had high fasting blood glucose levels with high average values of $137.9 \mathrm{mmol} / \mathrm{L}$. Similarly, high post prandial blood glucose levels were noticed among $85 \%$ of study patients with high mean values of 208.6 $\mathrm{mmol} / \mathrm{L}$. The mean duration for which patients have been diagnosed with diabetes was 12.27 years.

Table 4 shows that there is weak positive correlation between fasting and post prandial blood glucose levels with the periodontal health indicators. The values of periodontal health indicators do increase with higher values of fasting blood sugar levels, however, this increase is very small and thus, is statistically insignificant. Except for the plaque index, all the other periodontal health indicators have increased significantly with increase in post prandial blood glucose level.

All the periodontal health indicators worsen with increase in duration of diabetes. This worsening is periodontal indicators with duration of diabetes is statistically significant with gingival index, loss of attachment and probing depth. This implies that as patients suffer from longer periods of diabetes, there in higher values of gingival index, loss of attachment and probing depth. Thus, the current study implies that compared to single measure of blood sugar levels, it is the duration of diabetes which is significantly associated with periodontal health (Table 5).

Table 3: Diabetes status of the study participants $(n=86)$.

\begin{tabular}{|l|c|c|c|}
\hline \multicolumn{1}{|c|}{ Diabetes test } & Categories & $\mathrm{n}(\%)$ & Mean \pm S.D. \\
\hline Fasting Blood Sugar (mmol/L) & Within normal range & $17(19.8)$ & $137.9 \pm 48.01$ \\
\cline { 1 - 3 } & Out of normal range & $69(90.2)$ & $208.6 \pm 77.6$ \\
\hline $\begin{array}{l}\text { Post Prandial blood sugar } \\
\text { (mmol/L) }\end{array}$ & Within normal range & $13(15.1)$ & $73(84.9)$ \\
\hline Duration of Diabetes (in yrs) & Out of normal range & & $12.27 \pm 7.8$ \\
\hline
\end{tabular}

Table 4: Correlation of periodontal health indicators with blood sugar levels $(n=86)$.

\begin{tabular}{|l|c|c|c|c|}
\hline \multirow{2}{*}{\multicolumn{1}{c}{ Diabetes test }} & \multicolumn{2}{|c|}{ Fasting Blood Glucose level } & \multicolumn{2}{c|}{ Post Prandial Blood Glucose level } \\
\cline { 2 - 5 } & Correlation coefficient (R) & P value & Correlation coefficient (R) & P value \\
\hline Plaque Index & +0.006 & 0.957 & +0.024 & 0.824 \\
\hline Gingival Index & +0.039 & 0.720 & +0.016 & 0.016 \\
\hline Gingival Recession & +0.062 & 0.569 & +0.014 & 0.014 \\
\hline Loss of Attachment & +0.005 & 0.963 & +0.021 & 0.021 \\
\hline Probing Depth & +0.038 & 0.729 & +0.022 & 0.022 \\
\hline
\end{tabular}

Table 5: Correlation of periodontal health indictors with age of the individuals with diabetes patients. $(n=86)$

\begin{tabular}{|l|c|c|}
\hline \multicolumn{1}{|c|}{ Periodontal health indicators } & Correlation coefficient (R) & P value \\
\hline Plaque Index & +0.427 & $<0.001^{*}$ \\
\hline Gingival Index & +0.551 & $<0.001^{*}$ \\
\hline Gingival Recession & +0.430 & $<0.001^{*}$ \\
\hline Loss of Attachment & +0.516 & $<0.001^{*}$ \\
\hline Probing Depth & +0.479 & $<0.001^{*}$ \\
\hline
\end{tabular}




\section{DISCUSSION}

During the early insulin era, prior to 1922, several investigators assumed that all individuals with diabetes suffered from periodontal disease. Later on, high prevalence of periodontal disease among patients with relatively severe diabetes was observed. ${ }^{6}$

Earlier studies have shown that individuals with diabetes have more gingival inflammation than healthy control individuals. ${ }^{1}$ It has been observed that there is a increase in the prevalence and severity of the periodontal disease in patients with diabetes mellitus. ${ }^{5,6}$ Many previous studies have shown that individuals with controlled diabetes exhibit less severe periodontitis than the ones whose disease are not in control..$^{7-12}$

An interesting trend was observed as diabetes history longer than 10 years showed greater loss of periodontal structures. ${ }^{14}$ Further studies on this showed that it was not until the ages of 40 to 60 that the difference in the loss of attachment began to approach statistically significant. ${ }^{15-17}$ Chronological age of the individuals with diabetes was found to be more significant than the duration of diabetes as regards to the severity of periodontal disease. ${ }^{9}$ It was also noticed in one of the studies where in the age group 40 to 49 years old with long duration of diabetes showed significantly more probing depth of $>6 \mathrm{~mm}$ and alveolar bone loss. ${ }^{18}$ Thus with these observations it becomes significant to note the duration of diabetes with special consideration to age of the subject.

In regards to the children, several studies have suggested that the ones with diabetes have higher gingival index scores than individuals without diabetess. ${ }^{19,20}$ With all this evidence it is confirmed that irrespective of the duration of the disease there is higher prevalence of sites with periodontitis in individuals with diabetes.

The hospital runs a special diabetes clinic where substantial number of patients report every week. No such prevalence has been studied before in this population. Thus this cross sectional study was designed where age group between 20 to 70 years was selected to see the association between diabetes mellitus and periodontal disease with special consideration to poorly controlled diabetes mellitus.

\section{CONCLUSION}

The current study showed positive association between noninsulin dependent diabetes mellitus and periodontal disease. The current study implies that compared to single measure of blood sugar levels, it is the duration of diabetes which is significantly associated with periodontal health. However to make this study more significant a bigger sample size would be preferred. Also it would be preferred if HB1Ac could be recorded for each patient to see the association.

\section{REFERENCES}

1. Rylander H, Ramberg P, Blohme G, Lindhe J. Prevalence of periodontal disease in young diabetics. J Clin Periodontol. 1987;14:38-43.

2. Shlossman M, Knowler WC, Pettitt DJ, Genco RJ. Type 2 diabetes mellitus and periodontal disease. J Am Dent Assoc. 1990;121:532-6.

3. Emrich LJ, Shlossman M, Genco RJ: Periodontal disease in non-insulin dependent diabetes mellitus. J Periodontol. 1991;62;123-31.

4. Cohen DW, Friedman LA, Shapiro J, Kyle GC, Franklin S. Diabetes mellitus and periodontal disease: two-year longitudinal observations. Part 1. J Periodontol. 1970;41:709-12.

5. Wu T, Dorn JP, Donahue RP, Sempos CT, Trevisan M. Association of serum C-reactive protein with fasting insulin, glucose and glycosylated haemoglobin: the Third National Health and Nutrition Examination Survey, 1988-1994. Am J Epidemiol. 2002;155:65-71.

6. Sandler HC, Stahl SS. Prevalence of periodontal disease in a hospitalized population. J Dent Res. 1960;39:439-49.

7. Finestone AJ, Boorujy SR. Diabetes mellitus and periodontal disease. 1967;16:336-40.

8. Cohen DW, Friedman LA, Shapiro J, Clayton K, Franklin S. Diabetes mellitus and periodontal disease. Two year longitudinal observation. Part I. J Periodontol. 1970;41:709-12.

9. Cianciola, LJ, Park BH, Bruck E, Mosovitch L, Genco R.J. Prevalence of periodontal disease in insulin dependent diabetes mellitus. J Am Dent Assoc. 1982;104:635-60.

10. Ainamo J, Lahtinen A, Uitto VJ. Rapid periodontal distruction in adult humans with a poorly controlled diabetes mellitus. A report of two cases. J Clin Periodontol. 1990;17:22-8.

11. Benveniste R, Bixler D, Conneally PM. Periodontal disease in diabetics. J Periodontol. 1967;38:271-9.

12. Tervonen T, Knuuttila M. Relation of diabetes control to periodontal pocketing and alveolar bone level. Oral Surg Oral Med Oral Pathol. 1986;61:346-9.

13. Loe H. Periodontal disease. The sixth complication of diabetes mellitus. Diabetes Care. 1993;16:329-34.

14. Glavind, L, Lund B, Loe H. The relationship between periodontal state and diabetes duration, insulin dosage and retinal changes. J Periodontol. 1968;39:341-7.

15. Belting CA, Hiniker JJ, Dummet CO. Influence of diabetes mellitus on the severity of periodontal disease. J Periodontol. 1964;35:476-80.

16. Sznajder N, Carraro JJ, Rugna S, Sereday M. Periodontal findings in diabetic and nondiabetic patients. J Periodontol. 1978;49:445-8.

17. Wolf J. Dental and Periodontal conditions in diabetes mellitus. A clinical and radiographic study. Proc Finn Dent Soc. 1997;73:1-56.

18. Hugoson, A, Thorstensson, H, Falk H, Kuylenstierna J. Periodontal conditions in insulin-dependent diabetics. J Clin Periodontol. 1989;16:215-23.

19. Leeper SH, Kalkwarf KL, Strom EA. Oral status of controlled adolescent type I diabetes. J Oral Med. 1985;40:127-33.

20. Sandholm L, Swanljung O, Rytomaa I, Kaprio EA, Maenpaa J. Periodontal status of Finnish adolescents with insulin-dependent diabetes mellitus. J Clin Periodontol. 1989;16: 617-20. 